\title{
Predicting population abundance and age structure: testing theory with field data
}

\author{
James R. Weinberg \\ Woods Hole Oceanographic Institution, Biology Department, Woods Hole, Massachusetts 02543, USA
}

\begin{abstract}
Relatively simple demographic models can be used to predict abundance and agestructure of populations whose spatial boundaries can be delimited, especially if there is little dispersal of individuals beyond those boundaries. Because these conditions are generally not fulfilled by marine populations, researchers rarely attempt to predict abundance or structure of these populations. In this study, these predictions were made for the bivalve Gemma gemma using finite population growth rates and limiting age distributions associated with Leslie matrices. The field population is enclosed in a small bay in Rhode Island, USA. Dispersal to and from the bay is low because the clam's life cycle does not include planktonic stages. The data set analysed consisted of a time series of clam abundance from 1978 to 1983 , as well as age-specific survivorship and fecundity for each cohort present. Annual forecasts for June 1979, 1980 and 1981 deviated from observed values by only 12 to $17 \%$. The June 1982 forecast predicted a major population crash which did occur 1 mo later. By multiplying successive matrices, annual forecasts were made up to $5 \mathrm{yr}$ ahead. In 4 of 5 years predictions fell within $95 \%$ confidence intervals for mean observed abundance. Empirical age distributions fit expected distributions, derived from the theory of imprimitive or periodic matrices. This study's success at predicting major properties of the field population from the Leslie model suggests that (1) assumptions of the model were satisfied, (2) the population was in equilibrium state with the environment each year, and (3) the model was realistic enough to describe dynamics of this natural population.
\end{abstract}

\section{INTRODUCTION}

The primary goal of any forecasting strategy is to accurately predict magnitude of a variable at a specific time in the future. If the variable of interest is population size, then predictions might be used in making decisions in agriculture, to control the spread of a disease, or in a campaign to prevent extinction of a species.

Accurate forecasts can sometimes be derived by modelling the pattern of past fluctuations in the variable (e.g. Box \& Jenkins 1970, Mendelssohn 1981 , Stocker \& Hilborn 1981, Fogarty 1988) or by associating the variable with changes in other variables (e.g. Ulanowicz et al. 1982, Rasmussen \& Kalff 1987). Those approaches require little understanding of what caused the variable of interest to change. In contrast, an objective of population ecology is to predict abundance accurately, based on an understanding of the system of births, deaths, and the dispersal of individuals.

Predictions of abundance are made from mathematical models which vary in complexity. For example, complex metapopulation models are needed to describe species with extensive larval dispersal, linking patches of adult organisms (Paine \& Levin 1981, Iwasa \& Roughgarden 1985, Roughgarden \& Iwasa 1986). Other marine species, like the bivalve studied here, do not have dispersing larvae. In these cases, simpler models which consider only one patch of organisms might be adequate to predict population abundances accurately. An example of such a model is the Leslie matrix (Leslie 1945, 1948, Goodman 1967). Although it is fairly realistic because it considers agestructure, the model is not density- or time-dependent.

After populations pass through transient phases (Caswell \& Werner 1978), in theory they should acquire asymptotic or limiting properties determined by parameters in their Leslie matrices, if the characteristics of the individuals in the population and the environment do not change. For example, the finite population growth rate $(\lambda)$ and stable age distribution (SAD) are 2 asymptotic properties of the primitive or nonperiodic Leslie matrix (sensu Cull \& Vogt 1973). Imprimitive or periodic (sensu Cull \& Vogt 1973) Leslie matrices also exist and have slightly different limiting properties. Their asymptotic finite population growth rate will be $\lambda$, 
as for primitive matrices. However, their asymptotic population age distributions should no longer conform to the SAD. Instead, age distribution should vary from season to season with an annual period, and the average of all distributions measured within a given year should equal the SAD of that cohort (Cull \& Vogt 1973, 1974, Cull 1980). Imprimitive matrices arise for species which live in seasonal environments and whose reproduction is restricted to spring or summer. Their population sizes and age distributions oscillate in 'waves' (Bernardelli 1941, Lewis 1942). For annually breeding species, if the investigator sets up the Leslie matrix with age classes shorter than $1 \mathrm{yr}$, then the resulting matrix will be imprimitive (Cull \& Vogt 1973).

The purpose of this paper is to test whether actual population growth rates and age distributions of a marine bivalve, which has seasonal reproduction. behaved according to the limiting growth rates and age distributions predicted by the theory described above.

\section{STUDY ORGANISM}

The clam Gemma gemma (Totten) inhabits marine sandflats of the northern temperate zone of America. Females produce several broods, each of $<100$ offspring, during warmer months of each year (Sellmer 1967, Weinberg 1985). The clam is benthic and has no planktonic larval dispersal stage. Individuals are typically $<5 \mathrm{~mm}$ in shell length, and potentially live up to 4 yr (Bradley \& Cooke 1959, Sellmer 1967, Green \& Hobson 1970, Thompson 1982).

The population studied occurs in Little Narragansett Bay, Rhode Island, USA. It is geographically isolated from other Gemma gemma populations because the bay has a narrow entrance and no other populations are known to exist in the bay or along the bay's outer coastline. Maps and descriptions of the site are given in Weinberg $(1983,1985)$ and Weinberg et al. (1986).

The population was sampled from 1978 to 1983 , at least 4 times per year, which provided data on 8 cohorts. This sampling frequency was necessary to estimate accurately number and sizes of all age classes present throughout the year, particularly reproductive females and recruits. Samples provided a record of total population size through time (Fig. 1).

To compute Leslie matrices for each cohort, many variables were measured. Age-specific fecundity of each cohort was estimated from the positive relationship between female age, shell size and brood size (Weinberg 1985). Number of broods per female per year and sex ratio were estimated from dissections of specimens collected in the field and from laboratory studies with live clams (Weinberg 1985). Cohort-specific survivorship was measured directly from the reduction in density of

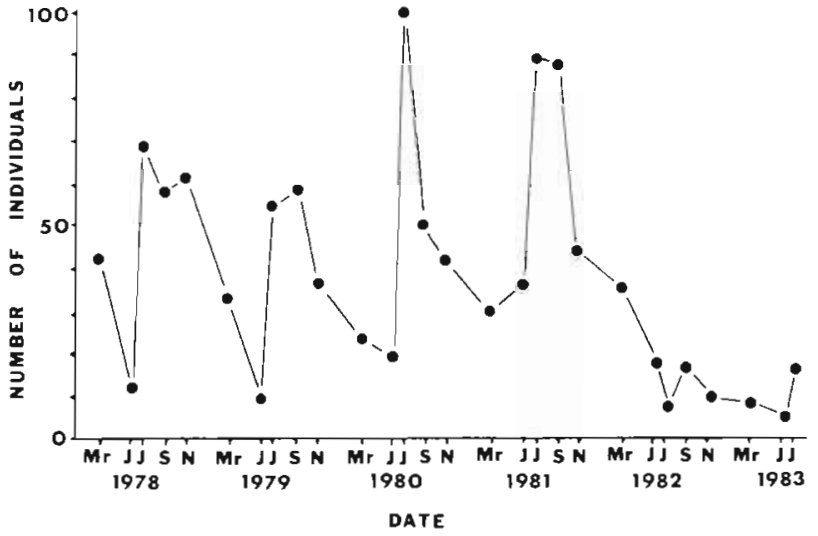

Fig. 1. Gemma gemma. Average number per $3.5 \mathrm{~cm}$ diameter core through time in the sampling plot ( 4 to 12 replicate samples per mean). $95 \%$ confidence intervals (CI) for means are given in Weinberg (1985). Lines connecting points are only to facilitate observation of pattern

each cohort with time. Complete Leslie matrices, divided into 3 mo age intervals, were computed for 4 cohorts, along with incomplete matrices on 4 other cohorts (life tables and matrices are given in Weinberg 1985. Weinberg et al. 1986). Due to annual periodicity of reproduction the resulting Leslie matrices were imprimitive.

Weinberg (1985) made the qualitative observation that cohort-specific growth rates from Leslie matrices were better predictors of population size changes from year to year than Box Jenkins time series models based only on total density. For example, the finite rate of growth, $\lambda_{i}$, where $i=$ year of birth, was close to $1[0.89$ to 1.20 ) for $3 y r$, and during that interval little change in total clam abundance occurred from 1 year to the next. After this stationary period (1978 to 1981 ) the population crashed from July densities of 50 to 100 individuals per $3.5 \mathrm{~cm}$ diameter core to about 7 individuals per core in July 1982. The theoretical growth rate at this time was $\lambda_{i}=0$, which implies an immediate crash. No attemps were made in Weinberg (1985) to quantify the accuracy of these annual forecasts or to check whether the model's assumption, that the average of the observed age distributions from each year fit the theoretical SAD, was fulfilled.

\section{METHODS}

To quantify forecast accuracy, annual predictions of population size $(\hat{N}(t))$ at time $t$ were made from the growth rate $\left(\lambda_{i}\right)$ for each cohort with complete life history data. These comparisons are not tautologous because predictions and actual measurements of population size were made from different information. Measurements of total population size were made directly by 
counting number of clams per core. In contrast, predictions of total population size were made by multiplying a total population size value from the past by $\lambda_{1}$, to predict a future, total population size. Computations of $\lambda_{i}$ involved data on the shell size, age, sex ratio, number of broods, fecundity per brood, and survivorship of clams in each cohort (i). Total population size was not one of the variables used to compute $\lambda_{\text {. }}$.

There were 2 time scales of forecasting: (1) year to year, and (2) multiples of $1 \mathrm{yr}$ using annually updated matrices, up to $5 \mathrm{yr}$ in the future. Within each year, the population was numerically dominated by the most recent cohort. Therefore, $\lambda$ of the dominant cohort was used to forecast total population size during that year. $\lambda_{i}=0.897,1.201,1.155$, and 0.0 for $\mathrm{i}=1978,1979,1980$, and 1981, respectively (from Weinberg [1985] and Weinberg et al. [1986]). Forecasting equations used to predict clam abundance 1 yr ahead were:

$$
\begin{aligned}
& \hat{N}\left(\text { June 1979) }=\lambda_{1978}^{4} N(\text { June 1978), }\right. \\
& \hat{N}\left(\text { June 1980) }=\lambda_{1979}^{4} N(\text { June 1979), }\right. \\
& \hat{N}\left(\text { June 1981) }=\lambda_{1980}^{4} N(\text { June 1980), }\right. \\
& \hat{N}\left(\text { June 1982) }=\lambda_{1981}^{4} N(\text { June 1981). }\right.
\end{aligned}
$$

Equations of the form:

$$
\hat{N}\left(\text { June year }_{\mathrm{p}+1}\right)=\left(\prod_{1978}^{\mathrm{p}} \lambda_{\mathrm{i}}^{4}\right) N(\text { June 1978), }
$$

where $p=1978,1979,1980,1981$, were used to predict populations size $1,2,3,4$, and 5 yr in the future. June 1978 was used as the initial population size for all forecasts here because it was the earliest observation from the start of a cohort year. Unlike the $\lambda$ values, this initial population size was fixed in the equation and never updated. Even though $\lambda_{1982}$ was not known, it was possible to compute an expected value for June 1983 (5 yr ahead), because $\lambda_{1981}$ was 0 .

Observed age distributions for the total population were plotted over time to examine whether waves such as those described by Bernardelli (1941) occurred, to see whether the age distributions from single sampling times ever approximated the SAD, and to statistically test whether the annual average distribution from 4 observed age distributions differed from the theoretical $\mathrm{SAD}$ associated with the numerically dominant cohort of the year.

\section{RESULTS}

\section{Prediction of population size}

In 1979, 1980 and 1981, annual forecasts were made over a wide range of population abundances and they deviated from observed values by 12 to $17 \%$ (Fig. 2). The 1982 observation date had a greater deviation

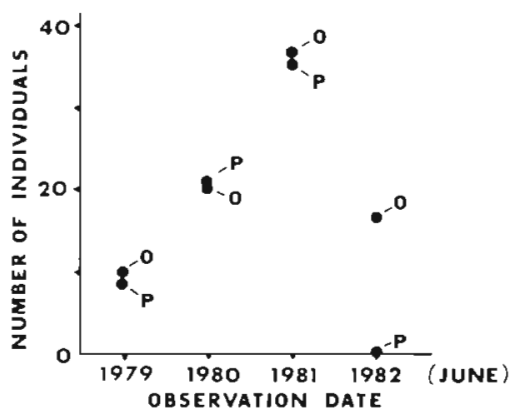

Fig. 2. Gemma gemma. Average observed (O) and predicted (P) clam abundance per $3.5 \mathrm{~cm}$ diameter core for $4 \mathrm{yr}$ of annual forecasts. Each forecast was computed from observed average density 12 mo earlier and survivorship and fecundity data for the numerically dominant cohort of that year

associated with it because the population did not crash as quickly as the model predicted. The deviation was due largely to the presence, in June 1982, of live individuals from the 1981 cohort. The population crash occurred shortly thereafter (Fig. 1) because most of these individuals failed to reproduce and then died by September 1982 (Weinberg 1985).

Because $\lambda_{i}$ was a good predictor of total population size 1 yr ahead, I also examined whether, given an initial population size, sequences of $\lambda_{j}$ 's could forecast years into the future. Deviations between observed and

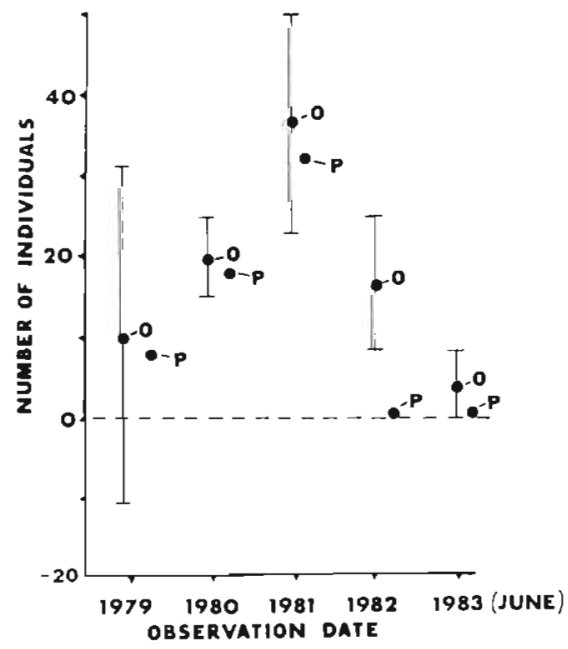

Fig. 3. Gemma gemma. Average observed $(\mathrm{O})$ and predicted (P) number of clams per $3.5 \mathrm{~cm}$ diameter sample for long-term ( $\geq 1$ yr) forecasting. $95 \% \mathrm{Cl}$ (from Weinberg 1985) are shown for observed means, based on $4,8,12,8$ and 8 samples, across years. Each $\lambda_{1}$ was used for 1 yr of forecasting with a multiplicative series starting from June 1978. (See 'Methods' for general forecasting equation)

expected population sizes (Fig. 3) were slightly greater than those in Fig. 2, because the initial value of $N(t)$ was set in June 1978 and was not updated after 12 mo, as it had been for $1 \mathrm{yr}$ ahead forecasts (Fig. 2). Yet, 
except for the 4 yr ahead forecast for June 1982 (Fig. 3), deviations were small enough that predicted values fell within independently derived $95 \%$ confidence intervals (from Weinberg 1985) for means for observed population sizes. Also note that the model's premature prediction of a population crash in 1982 and 1983 was realized by 1983 (Fig. 3).

\section{Age distributions}

In agreement with theory, observed age distributions of individuals never approximated the theoretical SAD of any cohort (e.g. Fig. 4). While the SAD's called for individuals in all age classes, actual population distributions typically consisted only of individuals of 2 or 3 ages, the newborn cohort and survivors of the 2 cohorts which

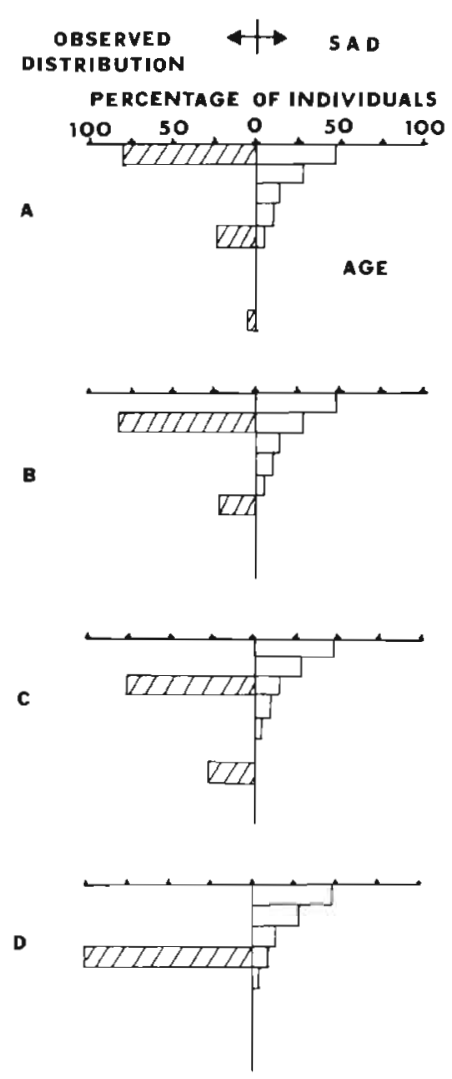

Fig. 4. Gemma gemma. Observed and expected (SAD) age distributions during 4 seasons of 1 yr (A) September 1979, (B) late November 1979, (C) early March 1980, (D) June 1980. Age classes are in 3 mo intervals starting from birth. Only the 1979 cohort's SAD and the observed data from September 1979 to June 1980 are presented. Data from other cohorts had similar patterns

preceded it (Fig. 4). The individuals moved from one age to the next with each survey date and produced the population waves described by Bernardelli (1941).
Unlike the individual age distributions, the average age distribution (Fig. 5) of the 4 distributions measured annually (Sep, Nov/Dec, Feb/Mar, Jun) was vегу close to theoretical SAD's for 1978, 1979, and 1980 data (contrast Fig. 4 with Fig. 5). The 1978 and 1980 cohorts

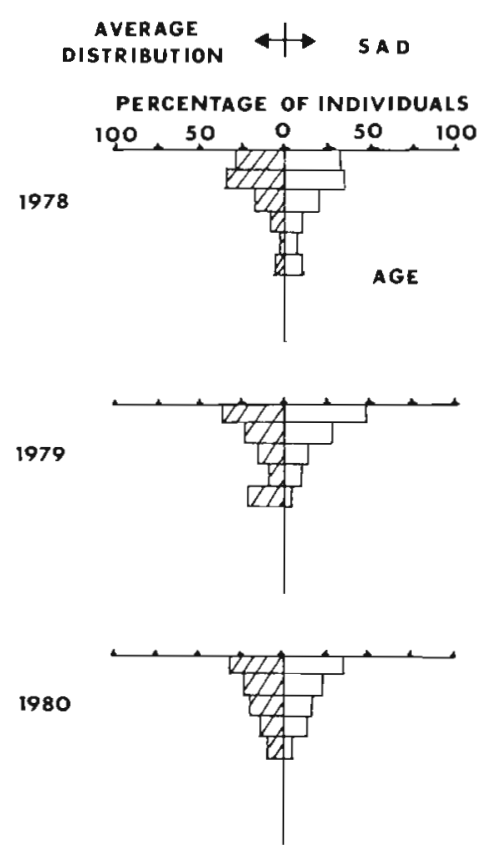

Fig. 5. Gemma gemma. Observed average age distributions and expected SAD's for 3 cohorts $(1978,1979,1980)$. Age classes are in 3 mo intervals starting from birth. Average distributions involve seasonal data collected between September 1978 and June 1981

average distributions did not differ significantly from their theoretical SAD's ( $\chi^{2}$ test, $p>0.05$ ). The 1979 cohort's distribution did differ from its SAD $(p<0.01)$ due to an excess of older individuals from the preceding cohort.

\section{DISCUSSION}

Forecasts were made for total population size in June of each year, based on the age-specific survivorship and fecundity schedule of the cohort which numerically dominated the population in the 12 mo preceding the forecast. Of 4 annual forecasts, 3 were within $17 \%$ of observed population sizes. The fourth forecast incorrectly predicted an immediate population crash; however, the prediction was actually premature rather than incorrect. When population growth rates were joined together in a multiplicative series, the model generated annual forecasts up to 5 yr ahead from an initial starting value in 1978. These forecasts fell within $95 \%$ confidence intervals of observed population sizes in 4 of 5 
years and were strikingly close to observed means. The one forecast which was outside the interval was the result of a premature prediction of the eventual crash.

Overall success of the Leslie model in forecasting was due to characteristics of the species studied which allowed precise measurement of important life history parameters. Brood size is typically $<100$, and can be counted precisely. Furthermore, offspring are born as shelled juveniles which immediately take up a benthic existence beside adults. Except for the few that might have been carried away by unusually strong currents generated by storms (Green \& Hobson 1970, Thompson 1982), all individuals could be accounted for because they remained at the study site.

The 4 cohorts analyzed, for which complete life table data were available, may be atypical of this species because they had short (1 to 2 yr) life spans. The presence of a few 3 yr old clams in my earliest samples along with sedimentary deposits, at the study site, of large Gemma gemma shells from past generations demonstrate that this species can live and reproduce for several years. Short life spans simplified population structure and improved forecast accuracy because forecasts of total population size were based only on the population growth rate of the numerically dominant cohort. If 3 or 4 cohorts had been abundant at the same time, methods used to forecast would have been inadequate because $\lambda_{i}$ of any one cohort could not represent the entire population. Thus, although G. gemma may be an ideal marine species for forecastig, the success seen here might not have occurred if individuals had lived longer.

There is evidence that intraspecific competition affects Gemma gemma growth and fecundity at the study site (Weinberg 1985). Why was the model able to produce accurate forecasts when it does not model density dependence in life history parameters? Density dependence was incorporated into my modelling indirectly because a different Leslie matrix was estimated for each cohort. As competition effects changed from year to year, parameters in matrices changed accordingly. No single matrix was used to forecast for more than $1 \mathrm{yr}$, and within each year, the population was modelled as growing exponentially at a rate determined by one Leslie matrix.

Population age distributions of Gemma gemma behaved in accordance with the theory of 'waves' (Bernardelli 1941) and the 'averaging theorem' (Cull \& Vogt 1973, 1974), which arise when a species has periodic reproduction separated by ages with no reproduction. Periodic reproduction translates into an imprimitive Leslie matrix (Cull \& Vogt 1974). One can convert an imprimitive matrix to a primitive one by pooling terms to reduce the number of age classes in the matrix (Leslie 1945). However, pooling is disadvantageous if it causes an error in computing $\lambda$ (Cull 1980), or if important aspects of the life history or population fluctuations being modelled are lost from consideration (Cull \& Vogt 1974). These later arguments apply here and justify use of the 3 mo age interval for modelling G. gemma's life history. As a consequence, predictions were based on the theory of imprimitive matrices rather than the more familar case. The population behaved according to the limiting properties of the model (growth rate and age structure), which suggests that the population was in its asymptotic phase and was not in a transient phase, as had been expected (Weinberg 1985).

Absence of a larval dispersal stage (and low migration in general) should, a priori, place Gemma gemma among species whose population sizes can be predicted successfully. Although more complex demographic models exist, the Leslie model was realistic enough in this case to achieve this goal.

Acknowledgements. I am grateful to H. Caswell, V. Starczak R. Zajac, and 3 reviewers for their critical comments on earlier drafts of this paper. $\mathrm{H}$. Caswell provided the computer program used to compute population growth rate and stable age distributions. I thank M. Dimmock for typing portions of the paper. This research was supported by EPA grant \# CR-814895-01-0, and by the Woods Hole Oceanographic Institution. This is W. H. O. I. Contribution No. 6560 .

\section{LITERATURE CITED}

Bernardelli, H. (1941). Population waves. J. Burma Res. Soc. 31 $1-18$

Box, F. E. P., Jenkins, G. M. (1970). Time series analysis, forecasting and control. Holden Day, San Francisco

Bradley, W., Cooke, P. (1959). Living and ancient populations of the clam Gemma gemma in a Maine coast tidal flat. Fish. Bull. Fish \& Wildlife Ser., No. 137, 58: 304-334

Caswell, H., Werner, P. A. (1978). Transient behavior and life history analysis of Teasel (Dipsacus sylvetris Huds.) Ecology 59: 53-66

Cull, P. (1980). The problem of time unit in Leslie's population model. Bull. math. Biol. 42: 719-728

Cull, P., Vogt, A. (1973). Mathematical analysis of the asymptotic behavior of the Leslie population matrix model. Bull. math. Biol. 35: 645-661

Cull, P., Vogt, A. (1974). The periodic limit for the Leslie model. Math. Biosci. 21: 39-54

Fogarty, M. J. (1988). Forecasting yield and abundance of exploited invertebrates. In: Caddy, J. F. (ed.) The scientific basis of shellfish management. J. Wiley \& Sons, New York, p. 701-724

Goodman, L. A. (1967). On the reconciliation of mathematical theories of population growth. J. R. statist. Soc. 130: $541-553$

Green, R. H., Hobson, K. D. (1970). Spatial and temporal variation in a temperate intertidal community, with special emphasis on Gemma gemma (Pelecypoda: Mollusca). Ecology 51: 999-1011

Iwasa, Y., Roughgarden, J. (1985). Evolution in a metapopulation with space-limited subpopulations. IMA J. Math. Appl. Med. Biol. 2: 93-107 
Leslie, P. H. (1945). On the use of matrices in certain population mathematics. Biometrika 33: 183-212

Leslie, P. H. (1948). Some further notes on the use of matrices in population dynamics. Biometrika 35 : 213-245

Lewis, E. G. (1942). On the generation and growth of a population. Sankhya 6: 93-96

Mendelssohn, R. (1981). Using Box-Jenkins models to forecast fishery dynamics: identification, estimation, and checking. Fish. Bull. U. S. 78: 887-896

Paine, R. T., Levin, S. A. (1981). Intertidal landscapes: disturbance and the dynamics of pattern. Ecol. Monogr. 5 (2): $145-178$

Rasmussen, J. B., Kalff, J. (1987). Empirical models for zoobenthic biomass in lakes. Can. J. Fish. Aquat. Sci. 44: 990-1001

Roughgarden, J., Iwasa, Y. (1986). Dynamics of a metapopulation with space-limited subpopulations.Theoret. Populat. Biol. 29: 235-261

Sellmer, G. P. (1967). Functional morphology and ecological life history of the gem clam, Gemma gemma (Eulamellibranchia: Veneridae). Malacology 5: 137-223

This article was submitted to the editor
Stocker, M., Hilborn, R. (1981). Short-term forecasting in marine fish stocks. Can. J. Fish. Aquat. Sci. 38: 1247-1254

Thompson, J. K. (1982). Population structure of Gemma gemma (Bivalvia: Veneridae) in South San Francisco Bay, with a comparison to some Northeastern United States estuarine populations. Veliger 24: 281-290

Ulanowicz, R. E., Liaquat Ali, M., Vivian, A., Heinle, D. R., Richkus, W. A., Summars, J. K. (1982). Identifying climatic factors influencing commercial fish and shellfish landings in Maryland. Fish. Bull. U. S. 80: 611-619

Weinberg, J. R. (1983). Population ecology of the marine bivalve Gemma gemma in relation to its infaunal community. Ph. D. thesis, University of Connecticut, Storrs

Weinberg, J. R. (1985). Factors regulating populations dynamics of the marine bivalve Germma gemma: intraspecific competition and salinity. Mar. Biol. 86: 173-182

Weinberg, J. R., Caswell, H., Whitlatch, R. B. (1986). Demographic importance of ecological interactions: how much do statistics tell us? Mar. Biol. 93: 305-310

Manuscript first received: August 23, 1988

Revised version accepted: January 20,1989 